\title{
Software para mejorar la aplicación de técnicas cuantitativas en estudios prospectivos
}

\author{
Software to improve the implementation of quantitative techniques in prospective studies \\ Software pour améliorer l’application de techniques quantitatives dans des études prospectives
}

Amaury Cabarcas Álvarez

Profesor Auxiliar y Coordinador de Autoevaluacion, Grupo de Investigación GIMÁTICA - Categoría B, Master of Science in Computer Engineering, Facultad de Ingeniería, Universidad de Cartagena, Cartagena - Colombia.

E-mail: acabarcasa@unicartagena.edu.co

Raúl J. Martelo Gómez Profesor Asistente y Jefe de Departamento de Redes y Telemática, Grupo de Investigación GIMÁTICA - Categoría B, Magister en Informática, Facultad de Ingeniería, Universidad de Cartagena,

Cartagena - Colombia.

E-mail: rmartelog1@unicartagena.edu.co

Luis C. Tovar Garrido

Profesor Asistente y Jefe de Departamento de Inteligencia Computacional, Grupo de Investigación GIMÁTICA - Categoría B, Magister en Ciencias Computacionales, Facultad de Ingeniería, Universidad de Cartagena, Cartagena - Colombia.

E-mail: Itovarg@unicartagena.edu.co

Artículo de investigación científica y tecnológica según clasificación COLCIENCIAS

Recepción: 19/02/2013

Corrección: $14 / 04 / 2013$

Aprobación: 05/05/2013

\section{Resumen}

Durante las dos últimas décadas se ha observado preocupación de organizaciones para lograr competitividad y obtener estabilidad en el mercado, impulsándolas a analizar ventajas de ir a un futuro deseado, haciendo uso de herramientas como los estudios prospectivos. Con el fin de optimizar la aplicación de estudios prospectivos, se han desarrollado herramientas de apoyo que aún no abarcan ciertos intereses de los involucrados como el uso de recursos económicos, ambientales, tecnológicos y humanos. Para suplir la necesidad encontrada, se planteó un software que contribuya al acompañamiento de estudios prospectivos apoyándose de tecnologías Web 2.0 independientemente de técnicas utilizadas por la persona guía del mismo, para lograr racionalizar recursos aprovechando las ventajas que ofrece la web. La investigación permitió concluir que los estudios prospectivos constituyen una alternativa viable para las organizaciones que desean planificar para alcanzar sus objetivos empresariales. Sin embargo, el intento de lograr el futuro deseable traería una serie de costos, que se incrementarán cuando la aplicación de estos estudios se lleve a cabo al margen de las herramientas, software y modelos adecuados. Por esta razón, el hombre debe emplear herramientas tecnológicas que combinen métodos, software, capacidad colaborativa y de integración como la ofrecida por la Web 2.0 para optimizar sus procesos y permitir así, que áreas como la prospectiva, alcancen un alto nivel de eficiencia y masificación.

Palabras clave: estudios prospectivos, tecnologías Web 2.0, ambientes organizacionales, matriz de impactos cruzados.

\section{Abstract}

Over the last two decades, organizations have expressed concern on achieving competitiveness and obtaining market stability, which has driven them to analyzing the advantages of going to the desired future by using tools like prospective studies. In order to optimize the use of prospective studies support tools were developed that still do not cover certain interests of stakeholders, including: use of economic, environmental, technological, and human resources. To supply the need identified, software was proposed to contribute to supporting prospective studies based on web 2.0 technologies, independent from techniques employed to optimize resources exploiting advantages offered by the Internet. This research concluded that prospective studies are a viable alternative for organizations seeking to achieve their business goals; however, the attempt to accomplish the desirable future will bring a range of costs, which are increased when the implementation of these studies conducted apart from tools, software, and models. For this reason, we must employ methods that combine technological tools, software, collaborative and integration capabilities as offered by Web 2.0 to optimize their processes and, thus, allow areas like prospective to achieve high levels of efficiency and overcrowding. 


\section{Résumée}

Pendant les deux dernières décennies on a pu observer la préoccupation des organisations pour atteindre la compétitivité et obtenir une stabilité dans le marché, ce qui les a obligés à analyser les avantages d’aller vers un futur désiré en utilisant des outils tels que les études prospectives. Dans le but d'optimiser l'application de ce type d'études, on a développé des outils de soutien qui ne comprennent encore certains intérêts des involucrés comme par exemple l'usage de ressources économiques, environnementales, technologiques et humaines. Pour satisfaire le besoin identifié, on a proposé un software qui contribue à l'accompagnement d' études prospectives, en s'appuyant sur des technologies Web 2.0 indépendamment de techniques utilisées par la personne qui le guide, pour pouvoir rationnaliser des ressources en profitant les avantages qui offre le web. La recherche a permis de conclure que les études prospectives constituent une alternative viable pour les organisations qui désirent planifier pour atteindre leurs objectifs. Néanmoins, l'essai pour acquérir le futur désirable implique une série de coûts qui augmenteraient au moment où l'application de ces études soie mise en place sans compter les outils, le software et les modèles corrects. Pour cette raison, l'homme doit utiliser des outils technologiques qui combinent des méthodes, du software, de la capacité collaboratrice et de l'intégration comme celle offerte par la Web 2.0 pour optimiser ses processus et permettre ainsi, que des aires telles que la prospective atteignent un haut niveau d'efficacité et de massification.

Mots clef: études prospectives, technologies Web 2.o, environnements organisationnels, matrice d'impacts croisés.

\section{Introducción}

Prospectiva se podría considerar como una forma razonada y asentada de obtener bases sólidas de cómo y cuál puede ser el futuro. Miklos \& Tello en su escrito "Planeación prospectiva: una estrategia para el diseño del futuro" (1991) sostienen que "la prospectiva consiste en actuar y concentrar la atención sobre el porvenir imaginándolo a partir del futuro y no del presente".

Al analizar el flujo de un estudio prospectivo se observan diferentes etapas. La primera etapa, corresponde a la presentación de la realidad, se debe realizar un diagnóstico de la situación tal cual es y de ser posible con información en relación con la historia o antecedentes existentes respecto al objeto de estudio, esto permite visualizar un futuro deseado (etapa 4). Para alcanzar este estado, es necesario hacer un análisis de recursos materiales, económicos y humanos con que se cuenta. Esta situación normalmente corresponde al "estado ideal" que en general no es fácil alcanzar y es necesario visualizar un futuro factible (etapa 3 ) y de acuerdo con los medios que se cuenten para alcanzarlo, poder seleccionar el futuro (etapa 5). Al trabajar con estas etapas en forma sucesiva y en algunas ocasiones paralelamente, se llega a la definición y estructuración del proyecto, cuya evaluación permite llegar a la etapa de decisión o acción (etapa 6).
Las etapas antes mencionadas, involucran utilización de algunos recursos como el personal, el tiempo, el espacio, el conocimiento, entre otros, que finalmente convierten un estudio prospectivo en un proyecto costoso y difícil de ejecutar de forma equitativa para cualquier organización; por consiguiente este documento tiene como fin mostrar la implementación de un software con las técnicas cuantitativas para el acompañamiento de estudios prospectivos organizacionales que permita racionalizar los recursos invertidos con el apoyo de tecnologías Web 2.0 de manera que se abarquen las etapas de un estudio prospectivo en un sistema integrador y lograr la masificación de las metodologías prospectivas en Colombia.

La importancia de la investigación, se basa en las limitantes observadas tanto en la realización de estudios prospectivos como en herramientas existentes diseñadas para realizar acompañamiento de los mismos, con lo cual se brindan tópicos que serán descritos y solucionados con la herramienta propuesta. Se espera que el lector conozca el aporte que se hace para la realización de la planeación estratégica de organizaciones a través de la herramienta propuesta comparada con el método tradicional para ejecutar estudios prospectivos.

\section{Metodología}

La metodología utilizada para el desarrollo del presente trabajo corresponde a la de investigación aplicada, porque parte de los conocimientos existentes, enfocándose en la utilización y consecuencias prácticas de conocimientos, los cuales son de gran utilidad para el estudio y solución de una problemática en el mundo empresarial.

Según las técnicas de recolección empleadas para responder al problema de investigación se define como de análisis documental. Rojas (2005), plantea que este tipo de investigación se caracteriza por el empleo predominante de registros gráficos y sonoros como fuentes de información, tales como enciclopedias, diccionarios y libros, artículos, revistas, tesis, informes técnicos, manuscritos, monografías, conferencias, catálogos y ensayos, entre otros, muchas de las cuales serán tenidas en cuenta para la obtención de la información que soporte la investigación del presente proyecto.

Resulta importante hacer énfasis en lo que Martelo Gómez, Peña Pertuz \& Tovar Garrido (2009) resaltan en su artículo donde se presentan una diversidad de técnicas dentro de los métodos de la prospectiva, que de acuerdo con la organización en estudio resultan más apropiados unas que otras. La primera técnica cuantitativa que se aplica pertenece al análisis estructural, que toma la organización u objeto de estudio, como un sistema conformado de un conjunto de elementos y relaciones entre sí. La estructura del sistema, es decir, la red de relaciones entre estos elementos, es esencial para comprender su evolución, porque la misma conserva cierta permanencia. 
El análisis estructural es un instrumento para clasificar las ideas que posibilitan la descripción de un sistema mediante una matriz que relaciona todos los elementos constitutivos y que a través de su estudio se evidencian las variables claves para la evolución del propio sistema. Medina (2000) lo define como un método de reflexión sistemática que otorga un buen peso a la intuición de partida permitiendo fortalecer el sentido común y la lógica del método. El objetivo del análisis es precisamente poner de relieve la estructura de relaciones entre las variables cualitativas, cuantificables o no, que caracteriza el sistema estudiado. Concretamente, ofrece la posibilidad de describir un sistema mediante el uso de una matriz que interconecta los componentes del sistema. Este método permite estudiar estas relaciones e identificar las variables esenciales.

El análisis estructural para el estudio prospectivo comprende varias etapas: la inicial es la identificación de variables. En la herramienta propuesta, se identifican tres roles: comercial (identifica cada proyecto, es decir, campo de acción donde se realiza el estudio prospectivo), un rol coordinador (pertenece a la persona que guía el proceso del estudio prospectivo) y un rol participante (cada participante se asocia con cada proyecto).

\section{Descripción del problema}

Medina y Ortegón (2006) plantean que la prospectiva es "una disciplina para el análisis de sistemas sociales, que permite conocer mejor la situación presente, identificar tendencias futuras y analizar el impacto del desarrollo científico y tecnológico en la sociedad", analizando factores de mayor importancia e impacto para la misma, como por ejemplo, para una empresa del sector comercial, el comportamiento del mercado y su participación en él, su posicionamiento frente a la competencia, además, de la satisfacción o inconformidad por parte de los clientes.

Según Olivé (2006), el análisis prospectivo debe ser un proceso estratégico, es decir, tratar de anticipar los retos que el futuro plantea y exponer un conjunto de decisiones encaminadas a cumplir los objetivos trazados previamente. La formulación de estrategias para el incremento de la competitividad en las empresas, se ha convertido en un elemento de apoyo vital para la construcción de un futuro exitoso a mediano y largo plazo. Sin embargo, gran cantidad de organizaciones aun no comprenden la necesidad de realizar estudios que logran lo que plantea Noguera \& Rincón (2008): “anticipación en vez de la reacción, evitando que el futuro arrastre lo que encuentra a su paso, y producir estímulos y datos en momentos donde ya no son de utilidad ni existe la posibilidad de elección”.

Las principales razones por las que las empresas se resisten a la aplicación de un estudio prospectivo, radican en altos costos que produce la realización del mismo, teniendo en cuenta que para la elaboración de talleres se recomienda efectuarlo en espacios diferentes a locaciones de la empresa, por lo cual se debe pensar en la contratación de un lugar con capacidad suficiente para albergar a todos los participantes. De igual forma, se debe presupuestar costos adicionales relacionados con el equipo durante los días que se desarrolle el taller.

Otra razón para considerar, es la ausencia del personal de sus lugares de trabajo en jornadas laborales, significando una reducción en las horas/hombre disponibles para la ejecución de las actividades dentro de la empresa. Además, el costo en papelería y contratación del experto quien dirigirá el taller, son solo algunos de los recursos que no pueden ser pasados por alto al momento de presupuestar un estudio prospectivo.

No obstante, se debe tener en cuenta que los costos y la participación dependen del contexto. Cada día es más frecuente realizar encuentros con gran número de participantes (más de 500 personas en algunos casos), que afecta de forma directa el tiempo que se debe invertir en la realización de los talleres, así como los recursos económicos requeridos.

En la mayoría de los talleres mencionados, de acuerdo con la técnica aplicada, para el caso cuando son cuantitativas, reflejan el hecho que para realizar valoraciones sobre relaciones se basan en consenso al que se llega con los participantes en el taller, producto de una discusión sobre los puntos de vista en la relación de variables involucradas, como es el caso del análisis estructural en la matriz de impactos cruzados. Al iniciar se toma el tiempo necesario para cada valoración, pero al ir desarrollando la matriz, factores como el cansancio de los participantes y el tiempo transcurrido, es limitado por el contrato que se tiene en el lugar donde se está realizando el estudio, el contrato con la persona que guía el proceso y los costos que incurren por parte de su organización y trae como consecuencia que se agilice y se asignen valores de manera no tan discutida como los iniciales, lo que podría repercutir al final de la aplicación de la matriz que se produzcan como variables esenciales que realmente no lo sean y viceversa.

Las razones anteriormente expuestas, son los argumentos con los que con frecuencia respalda una organización la negativa de inversión a un estudio, sin pensar a profundidad que probablemente estos costos no signifiquen mucho comparado con los que puede costarle una mala decisión en un futuro.

Teniendo en cuenta las situaciones mencionadas, se propone la implementación de un modelo apoyado en tecnologías Web 2.o, que funciona como herramienta de acompañamiento en la realización de estudios prospectivos con técnicas cuantitativas, contribuyendo de esta forma a racionalizar los recursos empleados.

Al utilizar la web como espacio de interacción, se permitirá vincular personas que estén físicamente en distintos lugares geográficos, minimizando los costos de movilización y manutención; asimismo, facilitará la realización de los talleres teniendo en cuenta la disponibilidad 
de tiempo del personal de la empresa, alcanzado de esta forma un ahorro significativo de recursos pertenecientes o brindados por la organización.

\section{Resultados y discusión}

\subsection{Herramienta software para estudios prospectivos aplicando técnicas cuantitativas}

Para realizar la prueba de la herramienta propuesta, se evaluaron los resultados obtenidos en la investigación empleando el marco conceptual de referencia a través de la implementación del escenario que será presentado como caso de estudio, donde se puede validar la aplicación y utilidad de la herramienta, para el acompañamiento de estudios prospectivos mediante la aplicación de tecnologías Web 2.0. El caso de estudio prospectivo es el comercio del archipiélago de San Andrés y Providencia, tomado del libro "La prospectiva y sus técnicas" de Miklos y Tello (1991).

Para dar inicio a un estudio prospectivo, se empieza por aclarar que el administrador o persona que guía el estudio crea el proyecto, es decir, un usuario en el rol comercial, como se muestra en la Figura 1.

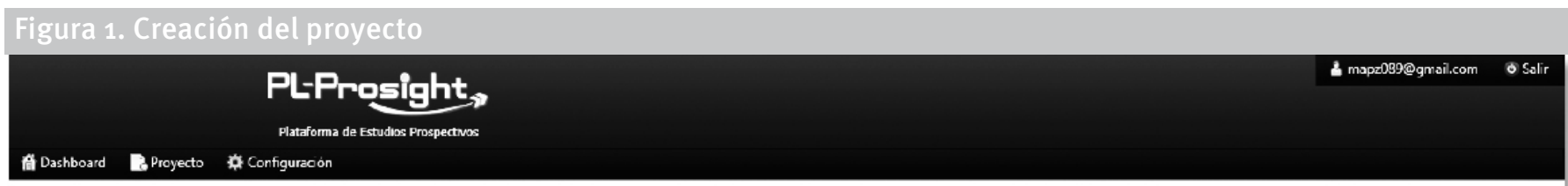

Proyecto

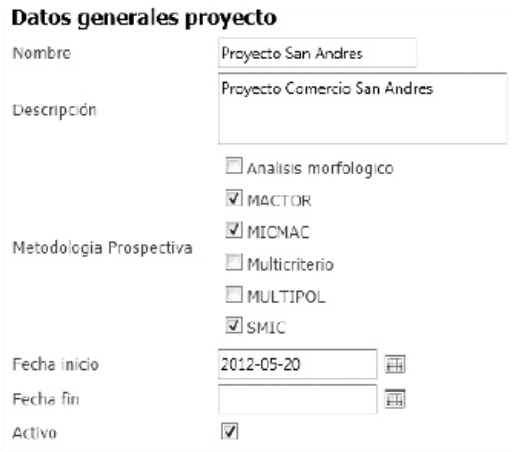

Datos generales coordinador

Primer Nombre Raul

Segunco Nombre

Primer Apellido

Segunco Apellido

Correo Electronico

Telefono

celular

Dirección

Activo

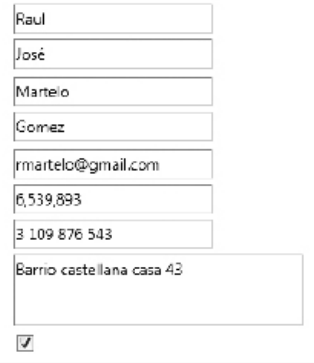

En la Figura 1 se observa cómo se puede asociar el coordinador a cada proyecto, el diligenciamiento de los datos del contexto del proyecto (empresa) además de especificar las técnicas a utilizar en dicho proyecto. Luego de haber creado el proyecto y el coordinador asociado con élque ya posee los privilegios de crear los participantes como se muestra en la Figura 2.

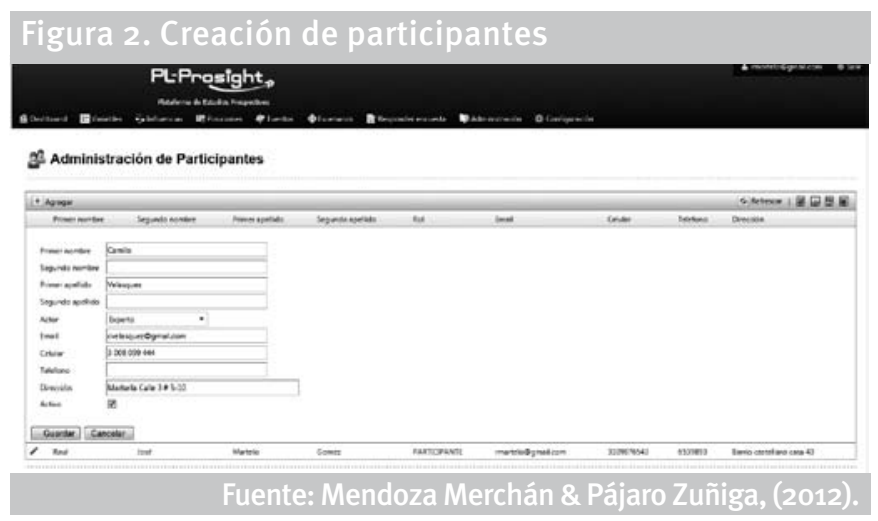

Para realizar la aplicación del estudio prospectivo en el caso tomado como escenario de prueba, se indica que se citó a 18 personas conocedoras del comercio de San Andrés, es decir a 18 expertos. Esta selección debe incluir a los cuatro agentes de desarrollo (el poder, la producción, el saber y la comunidad), a fin de evitar cualquier tipo de sesgo, porque cada quien se expresa con el discurso propio del sector al que pertenece.

Una vez que los expertos agotan la detección de problemas, se pasa a hacer una puesta en común de esa tempestad de ideas. Se procede anotar en un papelógrafo, los diferentes problemas que dictan los expertos. Como en muchos casos, más de un experto coincidió con la misma idea; esta situación se aprovechaba para precisar y enriquecer tanto el nombre del problema como su definición. El resultado sobre la tempestad de ideas de la problemática de San Andrés fue el siguiente:

Variables definidas. Resistencia a la tecnificación comercial, no diversificación comercial, saturación en la generación del empleo, no renovación tecnológica, bajo nivel cultural de la población, no planeación de la actividad económica, 
desconocimiento de los elementos básicos de mercadeo, desconocimiento del régimen legal del archipiélago, carencia de planeación a largo plazo, ausencia de líderes gremiales capacitados, carencia de orientación profesional, descoordinación entre la legislación nacional y la legislación del archipiélago, carencia de planeación a largo plazo, ausencia de líderes gremiales capacitados, carencia de orientación profesional, descoordinación entre la legislación nacional y la legislación del archipiélago, desmotivación de inversionistas, no aplicación de las políticas de fomento crediticio por parte de los bancos, desequilibrio entre el desarrollo socioeconómico y el desarrollo cultural, carencia de técnicos en diseño y evaluación de proyectos financieros, burocratización.

En consenso se definieron cada una de las anteriores variables, por ejemplo: Resistencia a la tecnificación comercial: Existe un criterio de continuar manejando las formas tradicionales de comercio. Se observa un marcado rechazo a la innovación y a la utilización de nueva tecnología como computador, formas preimpresas, entre otros.

Al utilizar la herramienta propuesta para realizar la inclusión de variables candidatas a ser evaluadas, se tiene lo siguiente al utilizar la variable Resistencia a la tecnificación comercial (Figura 3).

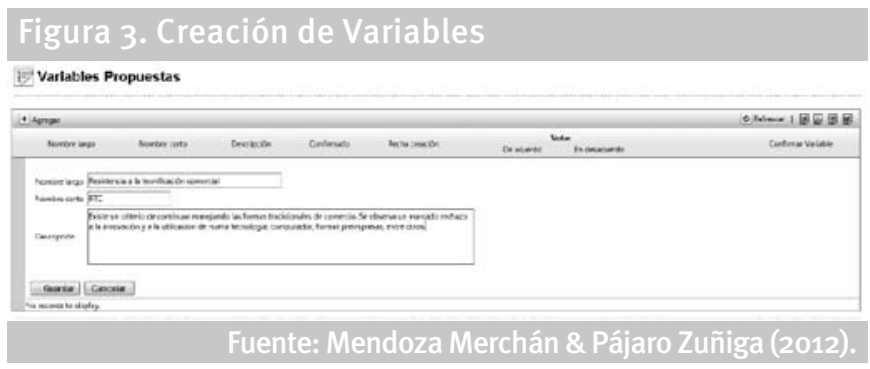

Al continuar el proceso anterior, se realiza el ingreso de cada variable con su definición. El valor agregado que genera la herramienta propuesta, frente a la manera como se realizan los estudios presenciales, es que se puede realizar consenso con objetividad a través de la valoración de la importancia que le dan los participantes a las variables y luego de este consenso el coordinador confirmará las variables que fueron seleccionadas como esenciales y surge la siguiente ventana en la herramienta propuesta (Figura 4).

\section{Figura 4. Valoración de Variables}

\section{iz Variables Propuestas}

\begin{tabular}{|c|c|c|c|c|c|c|c|c|c|}
\hline \multicolumn{7}{|c|}{+ Agregar } & \multicolumn{3}{|c|}{ (5) Refrescar I 图 圆圆 圈 } \\
\hline & & Nombre largo & $\begin{array}{l}\text { Nombre } \\
\text { corto }\end{array}$ & Descripción & Confirmado & $\begin{array}{l}\text { Fecha } \\
\text { creación }\end{array}$ & \multicolumn{2}{|c|}{ Votar } & Confirmar Variable \\
\hline > & 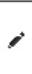 & $\begin{array}{l}\text { Resistencia a la } \\
\text { tecrificación comercial }\end{array}$ & RTC & $\begin{array}{l}\text { Existe un criterio de continuar manejando las formas tradicionales de comercio. Se } \\
\text { observa un marcado rechazo a la innovación y a la utilización de nueva tecnología: } \\
\text { computador, formas preimpresas, entre otros. }\end{array}$ & $\square$ & $\begin{array}{l}2012-05-21 \\
02: 28: 54 \\
\text { AM }\end{array}$ & 16 De acuerdo 3 & En desacuerdo 0 & confirmar variable \\
\hline > & 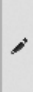 & $\begin{array}{l}\text { No diversificación } \\
\text { comercial }\end{array}$ & NDC & $\begin{array}{l}\text { Los renglones que se ofrecen siempre son lo mismos. El mismo comercio de } \\
\text { mercancías a turistos ya esta saturado. Este no se suplantado con alternativas que } \\
\text { puedan generar nuevas aplicaciones comerciales: como el proyecto de un astillero } \\
\text { para construir y vender barcos de mediano y pequefio calado. }\end{array}$ & $\square$ & $\begin{array}{l}2012-05-21 \\
02: 42.06 \\
\text { AM }\end{array}$ & [1) De acuerdo 0 & En desacuerdo 1 & Confirmar variable \\
\hline$>$ & 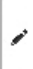 & $\begin{array}{l}\text { Saturación en la } \\
\text { generación del empleo }\end{array}$ & SAT & $\begin{array}{l}\text { No obstante ser el comercio el segundo generador de empleo ha llegado a un punto } \\
\text { en que es imposible expandirse y crear más fuentes de trabajo. }\end{array}$ & $\square$ & $\begin{array}{l}2012-05-21 \\
02: 42: 58 \\
\text { AM }\end{array}$ & 10 De acuerdo 0 & En desacuerdo 1 & confirmar variable \\
\hline$>$ & ; & No renovación tecnológica & NRT & $\begin{array}{l}\text { La tecnología empleada en el quehocer comercial es la clásica tradicional. Los } \\
\text { esquemas arcaicos se conservan y es muy poco lo que se innova o se transforma }\end{array}$ & $\square$ & $\begin{array}{l}2012-05-21 \\
02: 43: 20 \\
\text { AM }\end{array}$ & [t] De acuerdo 1 & En desacuerdo 1 & Confirmar voriable \\
\hline , & 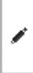 & $\begin{array}{l}\text { Bajo nivel cultural de la } \\
\text { población }\end{array}$ & BNC & $\begin{array}{l}\text { A pesar de la no existencia de analfabetismo según las ultimas cifras del Ministerio de } \\
\text { Educación en el archipiélago se hallan escolarizados solamente } 8.181 \text { alumnos en } \\
\text { educación básica primeria y } 2.007 \text { en educación básica secundaria-media vocacional. }\end{array}$ & $\square$ & $\begin{array}{l}2012-05-21 \\
02: 43: 54 \\
\text { AM }\end{array}$ & -1) De acuerdo 1 & En desacuerdo 0 & Confirmar variable \\
\hline$>$ & 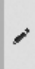 & $\begin{array}{l}\text { No planeación de la } \\
\text { actividad económica }\end{array}$ & NPA & Existe desorganización de la actividod económica. & $\square$ & $\begin{array}{l}2012-05-21 \\
02: 44: 12 \\
\text { AM }\end{array}$ & tit De acuerdo 0 & En desacuerdo 2 & confirmar variable \\
\hline$>$ & ; & $\begin{array}{l}\text { Desconocimiento de los } \\
\text { elementor básicos de } \\
\text { mercadeo }\end{array}$ & DME & $\begin{array}{l}\text { Se observa entre quienes manejan la actividad comercial el desconocimiento de los } \\
\text { procesos técnicos de identificación de demanda, previsión de la oferta y demás } \\
\text { condiciones del mercado modemo. }\end{array}$ & $\square$ & $\begin{array}{l}2012-05-21 \\
02: 44: 29 \\
\text { AM }\end{array}$ & (1) De acuerdo 2 & En desacuerdo 0 & Confirmar variable \\
\hline , & 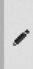 & $\begin{array}{l}\text { Desconocimiento del } \\
\text { régimen legal del } \\
\text { archipiélago }\end{array}$ & DLE & $\begin{array}{l}\text { San Andrés e islas tienen un régimen legal propio, cuyo desconocimiento traba el } \\
\text { funcionamiento de actividades económicas. En términos generales, quienes manejan la } \\
\text { economía de la isla desconocen este régimen legal. }\end{array}$ & $\square$ & $\begin{array}{l}2012-05-21 \\
02: 44: 45 \\
\text { AM }\end{array}$ & He acuerdo 1 & En desacuerdo 0 & Confirmar variable \\
\hline
\end{tabular}

Luego de incluir las variables al sistema, el siguiente paso consiste en la localización de las relaciones en la matriz del análisis estructural, que consiste en interrelacionar las variables en una matriz de doble entrada. La elaboración de la matriz es de forma cualitativa (la existencia o no de relaciones) pero también cuantificable. De esta forma se distinguen varias intensidades de las relaciones directas: fuerte, medias, débiles, potenciales o nula. El cumplimiento de la matriz se hace de dos maneras, por líneas, anotando la influencia de cada variable sobre las demás y/o por columna, anotando qué variables ejercen una influencia en cada varia- ble. La preparación de la matriz es una buena oportunidad para propiciar el diálogo, el intercambio y una reflexión que ayuda a crear un lenguaje común en el seno del grupo de análisis prospectivo, de modo que se favorezca a un consenso de forma más objetiva (Figura 5 ).

Para realizar lo anterior se procede a asignarle valor a cada variable y adicionar una justificación para cuando un participante observe el dato asignado por otro, pueda comprender las motivaciones que se tuvieron para realizar esa asignación; de esta manera se podría cambiar el punto de vista que se tenía, basándose en la explicación de un 


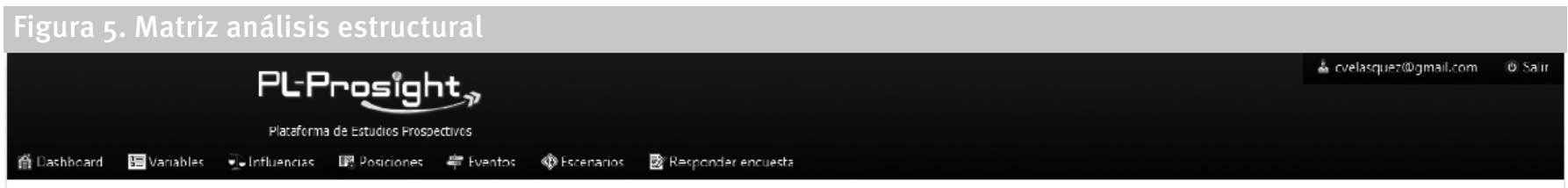

Matriz de influencias

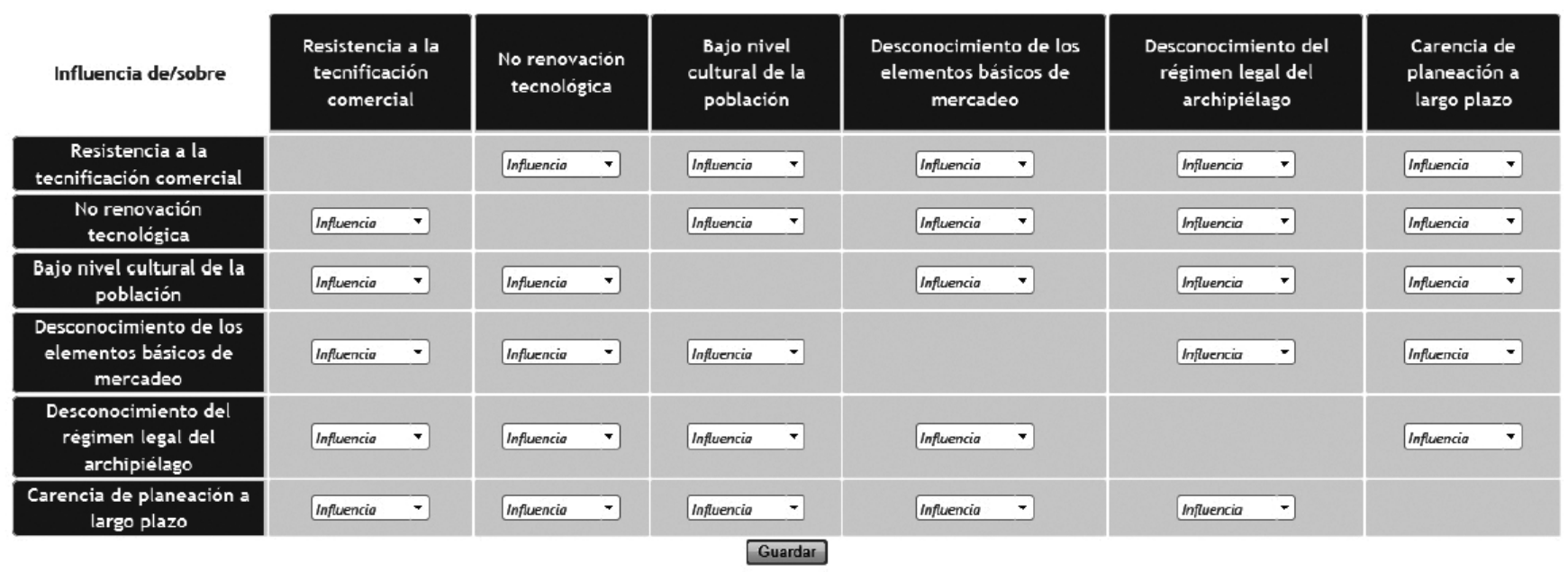

Fuente: Mendoza Merchán \& Pâjaro Zuñiga (2012).

participante que no se sabe exactamente quién es, lo que produce la eliminación de sesgos que se pueden presentar cuando se practica esta técnica con los asistentes de manera presencial, debido a que son grupos de trabajo con perfiles diferentes y muchas veces se cohíbe de realizar aportes objetivos por chocar con intereses de otros grupos de trabajo o tal vez existen relaciones de orden de mando organizacional entre los grupos de participantes. Luego de culminar el anterior proceso, en la ventana del coordinador se ve reflejada el total de cada relación de las variables, debido a todas las matrices valoradas de los participantes (Figura 6).

\section{Figura 6. Matrices Valoradas Participantes}

\section{Matriz de analisis estructural}

\begin{tabular}{|c|c|c|c|c|c|c|c|}
\hline Matriz de analisis estructural & Motricidad depen & ia directa & idad dependenc & directa & & & \\
\hline Influencia de/sobre & $\begin{array}{c}\text { Resistencia a la } \\
\text { tecnificación } \\
\text { comercial }\end{array}$ & $\begin{array}{c}\text { No } \\
\text { renovación } \\
\text { tecnológica }\end{array}$ & $\begin{array}{l}\text { Bajo nivel } \\
\text { cultural de } \\
\text { la población }\end{array}$ & $\begin{array}{l}\text { Desconocimiento de } \\
\text { los elementos básicos } \\
\text { de mercadeo }\end{array}$ & $\begin{array}{l}\text { Desconocimiento del } \\
\text { régimen legal del } \\
\text { archipiélago }\end{array}$ & $\begin{array}{l}\text { Carencia de } \\
\text { planeación a } \\
\text { largo plazo }\end{array}$ & $\begin{array}{l}\text { Motricidad } \\
\text { (total) }\end{array}$ \\
\hline $\begin{array}{c}\text { Resistencia a la } \\
\text { tecnificación } \\
\text { comercial }\end{array}$ & & 1 & 1 & 1 & 0 & $\mathbf{0}$ & 3 \\
\hline $\begin{array}{c}\text { No renovación } \\
\text { tecnológica }\end{array}$ & 1 & & 1 & 0 & 1 & 1 & 4 \\
\hline $\begin{array}{c}\text { Bajo nivel cultural de } \\
\text { la población }\end{array}$ & 1 & 1 & & 0 & $\mathbf{0}$ & $\mathbf{0}$ & 2 \\
\hline $\begin{array}{l}\text { Desconocimiento de } \\
\text { los elementos básicos } \\
\text { de mercadeo }\end{array}$ & 0 & 1 & 0 & & 0 & 1 & 2 \\
\hline $\begin{array}{l}\text { Desconocimiento del } \\
\text { régimen legal del } \\
\text { archipiélago }\end{array}$ & 0 & 1 & 1 & 0 & & 0 & 2 \\
\hline $\begin{array}{c}\text { Carencia de } \\
\text { planeación a largo } \\
\text { plazo }\end{array}$ & 1 & 1 & $\mathbf{0}$ & 0 & 1 & & 3 \\
\hline Dependencia (total) & 3 & 5 & 3 & 1 & 2 & 2 & 16 \\
\hline
\end{tabular}


Es de aclarar que la interpretación que se tiene con la Figura 6 es que la influencia es directa, cuando cualquier cambio en A modifica a B; es la resultante del juego de relaciones a corto y mediano plazo.

Cuando una variable influye sobre otra, directa 0 indirectamente, se puede decir que se encuentra la presencia de una influencia real o potencial; en caso que sea una constatación de lo que está sucediendo realmente, es una influencia real de las variables. Pero si esto no es lo que está aconteciendo en el momento presente, sino que se piensa que cierta variable debería influir sobre otra, a nivel del deber ser, esta influencia es potencial; por consecuente, integra relaciones que eventualmente nacerán después y no ejercerán su influencia en el sistema sino en el muy largo plazo. Cada variable lleva asociado un indicador de motricidad y dependencia. La motricidad es el porcentaje de influencia de cada variable, es decir el número de variables de las cuales influye, indica la fuerza que tenga cada una sobre las demás. Así, la variable de mayor motricidad es la más influyente, mientras que la de menor motricidad es la menos influyente.

Para una mejor visualización de las relaciones de motricidad y dependencia de variables, se grafican en un cuadro cartesiano que demarca cuatro zonas específicas, las cuales tienen las combinaciones de mayor a menor grado tanto de dependencia como de motricidad, para lo cual las variables ubicadas en el plano de mayor motricidad y dependencia serán donde se ubicarán los proyectos a realizar

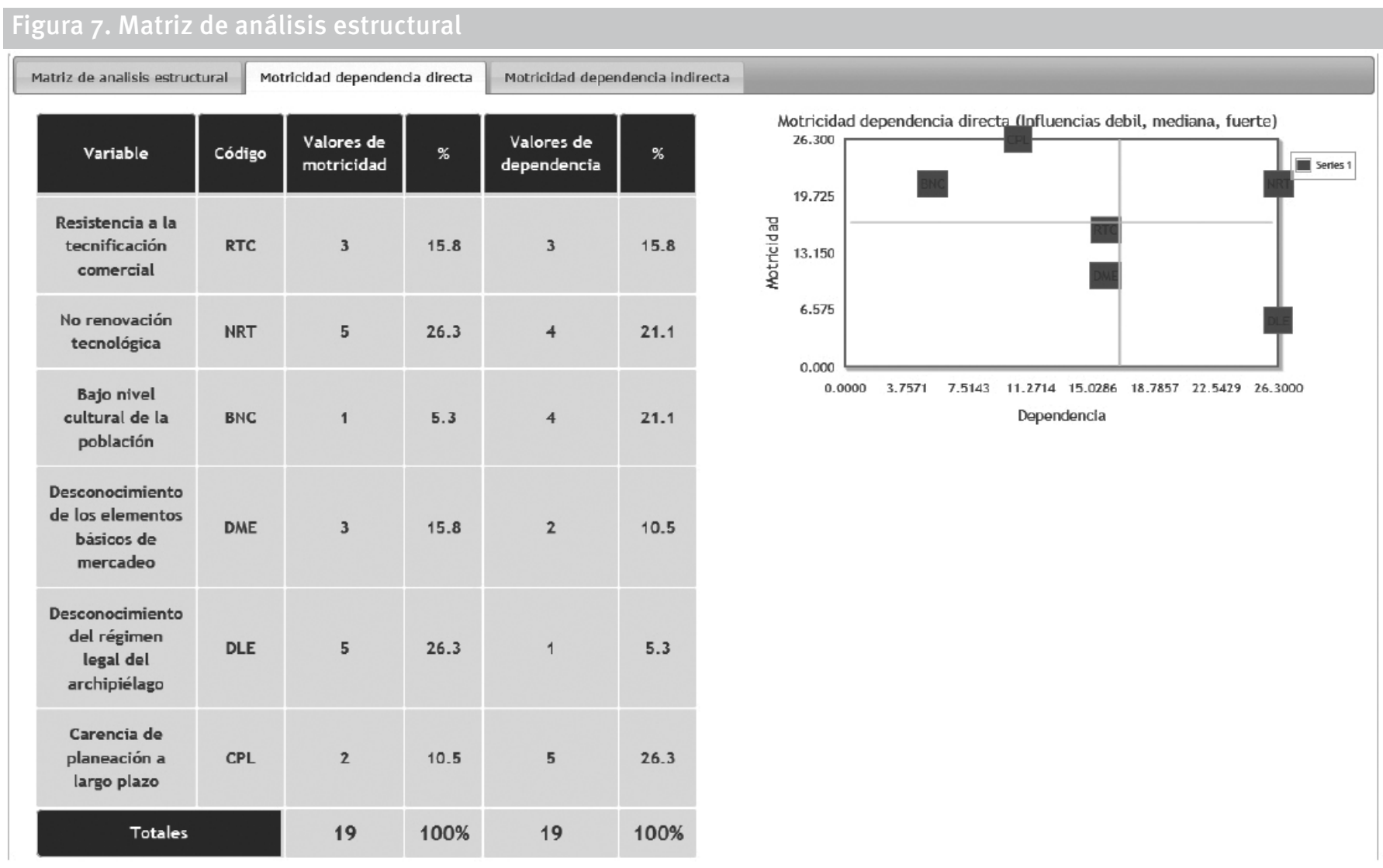

Fuente: Mendoza Merchán \& Pájaro Zuñiga (2012).

Lo anterior es el resultado del Método MICMAC, seleccionar que variables son las claves y entre ellas las importantes en cuanto a motricidad y dependencia, pues reflejan en qué se van a invertir para proyectos del sistema en estudio. Se pudo evidenciar que las variables claves resultantes en el estudio prospectivo realizado de manera tradicional fueron las mismas que se obtuvo con la aplicación de la herramienta, ellas son: resistencia a la tecnificación comercial, no renovación tecnológica, bajo nivel cultural de la población, desconocimiento de los elementos básicos de mercadeo, desconocimiento del régimen legal del archipiélago y carencia de planeación a largo plazo. Cabe resaltar que el modelo propuesto y el prototipo resultante están orientados únicamente al apoyo que brinda la herramienta para realizar estudios prospectivos, es decir, no abarcan la puesta en marcha y cumplimiento de las acciones detectadas como resultado del análisis. Además se cuenta con otras técnicas cuantitativas para el acompañamiento de estudios prospectivos como la extrapolación tendencias/análisis de impacto, indicadores/análisis de series de tiempo, bibliometría, análisis de patentes, modelación, delphi, votación y roadmaping. 


\section{Conclusiones}

Con la perspectiva del tiempo, se ha demostrado que la aplicación de un análisis prospectivo, a la luz de las múltiples aplicaciones en empresas y administraciones públicas, ha contribuido a estimular la reflexión estratégica colectiva y la comunicación en el seno de las empresas para mejorar la flexibilidad interna frente a la incertidumbre del entorno y a prepararse mejor para algunas posibles rupturas y a reorientar las decisiones en función de contextos futuros en los cuales sus consecuencias corren el riesgo de aparecer. Sin embargo, para que la aplicación de estos análisis prospectivos sea exitosa, es necesario la disposición de ciertos recursos como el espacio, el tiempo, el personal y el conocimiento, entre otros, que finalmente implican una significativa inversión monetaria.

Para contribuir a la racionalización de recursos mencionados antes, se desarrolló el presente proyecto enfocado en resolver la pregunta de investigación planteada inicialmente, donde para obtener una respuesta satisfactoria se llevó a cabo el análisis de los resultados de un caso real de análisis prospectivo siguiendo la forma tradicional de aplicación y luego se contrastaron con resultados del mismo ejercicio haciendo uso del prototipo resultante del modelo planteado en la presente investigación.

De este contraste realizado se pudo observar que al emplear un sistema basado en tecnologías Web 2.o para la realización de estudios prospectivos, se alcanzan ventajas que el modelo tradicional no contempla, tales como reducir el tiempo de discusión y realización del estudio, garantizar la disponibilidad e integridad de la información, permitir la interacción de diferentes expertos en la ejecución de un estudio sin requerir su presencia física, facilitar la masificación de técnicas prospectivas al representar un menor costo y consecuentemente mejorar la competitividad de organizaciones, brindando la oportunidad de crecer de forma equitativa, entre otros beneficios. Las ventajas obtenidas con el modelo y prototipo resultante mencionadas a lo largo del documento, evidencian y soportan que la consecución de objetivos del presente proyecto se llevó a cabo de forma satisfactoria.

Al contrastar los resultados obtenidos empleando el método tradicional de estudios prospectivos con los resultados alcanzados con la herramienta software propuesta, se puede establecer que:

* Los resultados obtenidos por los dos métodos coinciden, es decir, que este resultado permite garantizar el correcto funcionamiento del sistema y la adecuada aplicación de los pasos metodológicos del análisis estructural, que fue la técnica seleccionada para probar la herramienta; es de aclarar que el resto de las técnicas cuantitativas están funcionando correctamente.

* Pese a que los resultados finales arrojados por el estudio eran iguales, el tiempo de discusión empleado para realizar establecimiento de las variables, al igual que sus dependencias era mucho mayor aplicando el método tradicional, porque se sufrieron algunos escollos que extendían la discusión más de lo debido. Por otro lado, con la herramienta propuesta el tiempo para cada una de las fases puede ser controlado por el coordinador, estableciendo fechas límite para cada actividad.

De las ventajas que se pudieron observar con la ejecución del estudio prospectivo del comercio en San Andrés sobre la herramienta propuesta, se pueden destacar las siguientes:

* Eficiencia y velocidad en las respuestas presentadas por el prototipo en cualquiera de los módulos implementados.

* La posibilidad de hacer un análisis de sensibilidad por parte del operador al permitir hacer variaciones a los datos de entrada y obtener diferentes resultados.

* Permite exportar resultados a documentos en formato de Microsoft Word, Excel y XML.

* Permite tener un historial de los estudios prospectivos realizados, porque cada estudio y su respectiva información pueden ser almacenados en una base de datos integrada a la aplicación principal.

La herramienta presentada además de cumplir con las limitantes identificadas en la descripción del problema, presenta las siguientes ventajas en comparación con las existentes en el mercado para el acompañamiento de estudios prospectivos:

Instalación Stand Alone. Estas herramientas no posibilitan ningún tipo de conexión múltiple que les permita compartir la información, por lo cual sólo podrá ser accedida por la persona encargada de coordinar el estudio para introducir la información. Es decir funciona en cada equipo instalado por sí solo.

Limitaciones regionales. Las herramientas mencionadas se encuentran disponibles únicamente en francés, creando una gran restricción para las personas o entidades de los países latinos o de habla hispana que deseen acceder a la aplicación de estudios prospectivos. La propuesta independientemente del idioma, puede realizar el acompañamiento al ofrecer vistas en cualquier idioma.

Resguardo de la información. Las herramientas enunciadas no cuentan con un sistema de base de datos para el manejo y respaldo de la información o datos suministrados, debido a que funcionan con un sistema de archivos que almacenan la información en formato XML y que debe ser cargado cada vez que se desee abrir el proyecto para visualizar la información, exponiendo de esta forma los datos a modificaciones no autorizadas 0 pérdida total.

Conectividad nula. Estas herramientas no poseen ningún tipo de conexión web que permita compartir la información en tiempo real, por lo cual resultados finales 
- parciales no podrán ser visualizados desde ningún lugar diferente al equipo donde se encuentre instalada la herramienta.

A nivel tecnológico se resaltan las siguientes ventajas: Disponibilidad Web. Al ser un sistema desplegado en Internet, garantiza que podrá ser accedido desde cualquier medio electrónico que cuente con una conexión web en cualquier lugar del mundo y en cualquier horario, permitiendo llevar a cabo un estudio sin tener en cuenta las limitaciones geográficas.

* Multilingüe. Al ser desarrollado empleando tecnologías de codificación que contemplan la traducción como una necesidad de globalización, el sistema ofrece la ventaja de realizar un estudio prospectivo sin limitaciones de idioma, permitiendo invitar personal que maneje un idioma u otro, enriqueciendo aún más la participación y puntos de vista con personal capacitado proveniente de cualquier país.

* Uso de nuevas tecnologías. Se ha desarrollado utilizando tecnología actualizadas tales como .NET, jquery, CSS, sindicación RSS, entre otros, que aseguran que el tiempo de obsolescencia de la aplicación sea en un tiempo mayor.

* Completo control y auditoría sobre las acciones. Para garantizar la confiabilidad de la información, todos los cambios que se lleven a cabo en la aplicación son registrados en la base de datos indicando el usuario y la fecha en que se realizó alguna modificación a la información. De manera, que si en un momento dado exista alguna inconsistencia, se cuente con los datos suficientes para rastrear el origen.

* Control de acceso por usuarios y permisos. La seguridad de la información debe ser garantizada, de allí que el sistema requiera el registro de un usuario para poder ser accedido. Adicionalmente, los menús o acciones son restringidos dependiendo de la función que haya sido asignada a un usuario determinado, dando permisos para llevar a cabo acciones específicas, tales como la generación de un reporte especial o la obtención de los resultados finales, entre otros.

* Manejo de reportes en múltiples formatos. Los resultados de un estudio prospectivo son la materia prima con la que la organización empezará a guiarse, de allí que se ratifique la importancia de poder manejar estos resultados en múltiples formatos con información oportuna y productiva. Dentro del sistema se puede obtener la información en XML, PDF, CSV, Excel, Word, tablas y gráficos.

* Constante actualización. Una aplicación al hacer uso de nuevas tecnologías es factible que permanezca en desarrollo y por tanto se requiera liberar frecuentemente versiones que contengan mejoras para el funcionamiento del sistema.
A nivel de proceso se resaltan las siguientes ventajas:

* Invitaciones a expertos. Brinda la posibilidad de extender la invitación de un estudio prospectivo a un nuevo participante en calidad de experto conocedor de la temática y facilitar su participación al no requerir su presencia física, sino únicamente su disponibilidad de colaborar vía web.

* Manejo de anonimidad. Los participantes en un estudio prospectivo podrán tener la seguridad que su información personal no será divulgada bajo ninguna circunstancia, así como el autor de las opiniones que hayan sido expresadas como parte del análisis de la problemática. Esto se desarrolló con el fin de aminorar la presión experimentada durante las valoraciones, evitando preocupaciones por el punto de vista expresado y sesgos que se ocasionan por jerarquías de mando.

* Transparencia en el proceso. Ofrece a participantes la posibilidad de consultar la información en tiempo real, contribuyendo a que se aumente la confianza en el proceso y se eviten posibles inseguridades en cuanto a la manipulación de la información.

* Integración de varios métodos prospectivos. A diferencia de herramientas existentes en el mercado, la presente solución ha sido diseñada de forma que se permita la aplicación de un estudio empleando diferentes métodos seleccionados.

* Reúne la funcionalidad de diferentes herramientas. El desarrollo de este prototipo logró esquematizar en un mismo lugar las herramientas necesarias para llevar a cabo un estudio prospectivo hasta la etapa de generación de escenarios, disminuyendo el tiempo que duraría la realización del mismo haciendo uso de diferentes aplicaciones.

* Base de conocimiento de participantes. Al manejarse un registro de usuarios de forma sistematizada, se cuenta con la información de participantes que manejan una temática específica y que podrían ser invitados a otro estudio prospectivo si ellos lo desean.

* Reducción de tiempo en discusiones. Los participantes realizarán sus aportes de forma electrónica, lo cual permitirá obtener respuestas más concisas y que puedan ser delimitadas rápidamente. De esta forma, se evitará caer en el escollo de desviar discusiones y extenderse más de lo debido.

* Reducción de papelería. Una de las principales ventajas que ofrece la tecnología web, es el registro de información que no requiere ser impresa para poder ser visualizada y actuar como soporte. De esta forma, el sistema minimiza la papelería empleada en talleres de prospectiva y por ende contribuye directamente a la reducción de la tala de árboles para la producción del papel. 
* No requiere movilización. Con el sistema PL-PROSIGHT se minimiza el número de reuniones presenciales que realizan los participantes, porque el espacio de interacción y colaboración es la web, con lo cual se evita la contratación o alquiler de un espacio específico durante largo tiempo para la realización del estudio.

\section{Recomendaciones}

Para complementar, una posibilidad de expandir esta herramienta, consiste en integrar otras metodologías de la caja de herramientas de la prospectiva estratégica como son las técnicas cualitativas, útiles para explorar el campo de los posibles y reducir la incertidumbre. Estas adiciones permitirían conformar una herramienta de apoyo completa que ofrecería variedad de alternativas de análisis a grupos prospectivos, al poder contar con las técnicas cualitativas y cuantitativas.

Actualmente la herramienta fue desarrollada para funcionar con los roles de comercial y participante/ coordinador, donde se ha abarcado la funcionalidad planteada a lo largo del documento. Sin embargo, existe una función que debe ser tenida en cuenta para un próximo desarrollo, el cual corresponde al rol administrador, quien es la persona encargada de configurar ciertos parámetros técnicos para el funcionamiento del sistema y por ende proyectos que en él se realizan.

Finalmente, el sistema resultante contiene una extensa documentación, que debe ser tenida en cuenta al momento de realizar la aplicación de un estudio prospectivo, porque fue diseñada específicamente para orientar la creación y desarrollo del mismo y de no considerarla puede abrir paso a posibles inconvenientes.

\section{Referencias}

Ackoff, R. (1973). Méthodes de planification de l' entreprise. Editions d' Organisation. Recuperado 20/02/2013 de: http://www.cnam.fr/servlet/com.univ.collaboratif. utils.LectureFichiergw?ID_FICHIER $=1295877017760$ http://www.cnam.fr/servlet/com.univ.collaboratif.utils. LectureFichiergw?ID_FICHIER $=1295877017760$

Castañeda, L. (2007). Software social para la escuela 2.0: más allá de los Blogs y las Wikis. Buenos Aires, Argentina: Universidad Tecnológica Nacional.

Cerezo, J. (2007). Web 2.o, una guia imprescindible para todos aquellos que quieren pasar a ser parte de las "multitudes inteligentes". Madrid, España: ESIC.

Colombia Ciencia y Tecnología (Colciencias). (2006). Prospectiva en la escena colombiana. Colciencias, 24 (1-2) (pp. 11-15).

Convenio Andres Bello (2004). Políticas, estrategias y consensos de acción en ciencia y tecnología de los países del Convenio Andrés Bello. Bogotá, Colombia: Editorial Nomos.

Decouflé, A. (1980). La prospective. Paris, Francia: Presses Universitaires de France.

Espinoza, J. (2008). La prospectiva territorial: Un camino para la construcción social de territorios de futuro. Bogotá, Colombia: Universidad Externado de Colombia.
Gargiulo, G. (2001). Prospectiva tecnológica en Argentina. Revista Científica de UCES, 5 (1) (pp. 43-55).

Godet, M. (1993). De la anticipación a la acción. Barcelona, España: Marcombo S.A.

Godet, M. (2000). La caja de herramientas de la prospectiva estratégica. Paris, Francia: Gerpa, Electricité de France, Mission Prospective.

Jouvenel, B. (1964). El arte de la conjetura. París, Francia: Editions du Rocher.

Jouvenel, H. (2004). Invitación a la prospectiva. Lima, Peru: Futuribles-CONCYTEC.

Kruchten, P. (1995). Planos Arquitectónicos: El Modelo de 4+1 Vistas de la Arquitectura del Software. IEEE Software, 12 (6) (pp. 42-50)

Marín, J. (2006). Web 2.o, una descripción muy sencilla de los cambios que estamos viviendo. La Coruña, España: Netbiblo.

Martner, G. y Köppen, E. (1989). Prospectiva y futurología en América Latina. Mexico D.F., México: Centro de Investigaciones Interdiciplinarias en Humanidades - Universidad Nacional Autónoma de México.

Medina, J. (1999). La prospectiva humana y social: alternativa de la nueva generación para América Latina. Santiago de Chile, Chile: Instituto Latinoamericano y del Caribe de Planificación Económica y Social.

Medina, J. (2006). Visión compartida del futuro. Cali, Colombia: Universidad del Valle.

Medina, J. y Mojíca, F. (2009). La prospectiva en Colombia Antecedentes, lecciones y desafios. En: Dos Santos, D. y Fellows, L. Prospectiva en América Latina: Evolución y desafíos (pp. 143-183), Bauru, Brasil: Canal 6.

Medina, J. y Ortegón, E. (2006). Manual de prospectiva y decisión estratégica: bases teóricas e instrumentos para América Latina y el Caribe. Santiago de Chile, Chile: Naciones Unidas.

Medina, M. (2000). Futurica: Prospectiva en acción. Caracas, Venezuela: Ediciones IESALC/UNESCO.

Mendoza-Merchan, L. y Pajaro-Zuñiga, M. (2012). Implementación de un modelo para el acompañamiento de estudios prospectivos organizacionales empleando tecnologias Web 2.o. Tesis de Grado Ingeniería de Sistemas. Universidad de Cartagena. Programa de Ingeniería de Sistemas. Cartagena, Colombia. [Tesis no Publicada].

Miklos, T. \& Tello, M.E. (1991). Planeación prospectiva: una estrategia para el diseño del futuro. Mexico D.F., México: Centro de estudios prospectivos: Fundación Javier Barros Sierra.

Miklos, T. y Tello, M. E. (1997). Planeación prospectiva. Mexico D.F., México: Limusa Noriega Editores.

Mojíca, F. (1991). La prospectiva: Técnicas para visualizar el futuro. Bogotá, Colombia: Legis Fondo Editorial.

Mojíca, F. (2002). Teoría y aplicación de la prospectiva: El futuro del comercio de flores colombianas en los Estados Unidos. Bogotá, Colombia: Universidad Externado de Colombia.

Mojíca, F. (2010). Francisco José Mojíca. The Millennium Project. Global Futures Studies \& Research. Nodo Colombiano. Recuperado 18/06/2010 de: http://www.franciscomojica.com/

Noguera L.M. y Rincón de-Parra, H. (2010) Costos de producción en las empresas del sector de la construcción:un caso de análisis prospectivo. Visión gerencial, 8, (pp. 107-120). Recuperado 25/09/2010 de: http://www.saber.ula.ve/ bitstream/123456789/31344/1/articulo8.pdf 
Observatorio de Prospectiva Científica y Tecnológica (OPCyT). (2000). Ejercicios Nacionales de Prospectiva. Recuperado 18/o9/2010 de: http://www.opcyt.setcip.gov.ar/la_prospectiva_6.htm

Olivé, E. (2006). Los estudios de futuro, herramienta para la competitividad de las empresas. La Habana, Cuba: Ministerio de Ciencia, Tecnología y Medio Ambiente.

OPTI. (1999). Primer informe de prospectiva tecnológica industrial. Futuro tecnológico en el horizonte de 2015 . Madrid, España: OPTI.

Pérez, M. (2005). ¿Qué es la prospectiva? Del mito a la realidad sobre la construcción del futuro. Recuperado 20/09/2010 de: http://ciid.politicas.unam.mx/semprospectiva/ dinamicas/r_estudiosfuturo1/revista/numero\%203/construf/conspira/wong.htm
Prospectiva 2020. (s.f.). Prospectiva 2020. Recuperado 18/02/2010 de: http://www.prospectiva2020.com/tipo. php?subsec $=14 \& \sec =3$

Ramos, A. (2002). Informe a Presidencia Programa de Prospectiva Tecnológica Uruguay 2015. Montevideo, Uruguay: Organización de las naciones unidas para el desarrollo industrial ONUDI.

Rincón, S. y Mujica, N. (2006). Estudio prospectivo de la gestión tecnológica en las empresas del sector metalmecánico del estado Zulia, Venezuela. Revista Venezolana de Gerencia, 9 (26) (pp.1-33).

Rivas, R. (2004). La prospectiva en el ámbito de las organizaciones. Lima, Peru: Pontificia Universidad Católica del Perú.

Rojas, R. (2005). Guia para realizar investigaciones sociales. Mexico D.F., México: Plaza y Valdéz.

Villarroel, Y., Popper, R. y Lozano, A. (2010). Prospectiva en América Latina. Una visión sistémica en espacios de catástrofe. Pensamiento divergente, I (1) (pp.59-91).

Cuadernos de Administración / Facultad de Ciencias de la Administración / Universidad del Valle Periodicidad: semestral / ISSN impreso Nº 0120-4645- ISSN electrónico N²256-5078 / Nombre abreviado: cuad.adm. Edición Vol. 29 N 49 (enero - junio de 2013)

Software para mejorar la aplicación de técnicas cuantitativas en estudios prospectivos/Amaury Cabarcas Álvarez, Raúl J. Martelo Gómez y Luis C. Tovar Garrido 